\title{
Reintroduction of Diazoxide after Diagnosis of Pulmonary Hypertension in a Patient with Transient Hyperinsulinism
}

\author{
Bahareh Schweiger $^{1}$ Pedro A. Sanchez-Lara ${ }^{1}$ Dor Markush ${ }^{2}$ Pooja Nawathe ${ }^{1}$ \\ 1 Pediatrics Department, Cedars-Sinai Hospital, Los Angeles, \\ California, United Sates \\ ${ }^{2}$ Guerin Congenital Heart Program, Smidt Heart Institute, Cedars- \\ Sinai Medical Center, Los Angeles, California, United Sates \\ Address for correspondence Bahareh Schweiger, DO, MPH, Cedars- \\ Sinai Hospital, 8700 Beverly Blvd Los Angeles, CA 90048, United Sates \\ (e-mail: bahareh.schweiger@cshs.org).
}

J Child Sci 2021;11:e80-e82.

\begin{abstract} Our case describes the reintroduction of diazoxide despite life-threatening pulmonary hypertension in our infant due to lack of therapeutic options for congenital

Keywords hyperinsulinism.

- congenital hyperinsulinism

- diazoxide

- pulmonary hypertension

- chlorothiazide

- side effects
\end{abstract}

\section{Introduction}

A 37-day-old 35-week premature infant with normal APGARs at birth presented to the endocrinology clinic for follow-up after being on diazoxide $13 \mathrm{mg} / \mathrm{kg} /$ day divided every 8 hours for hyperinsulinism (HI). On presentation to the clinic, a rapid response code was called as the patient appeared cyanotic and had abdominal distension and suspected necrotizing enterocolitis. He was urgently intubated for impending respiratory failure. Chest radiograph showed cardiomegaly and mild pulmonary edema. The initial echocardiogram showed a small ductus arteriosus with exclusive right-to-left shunting consistent with severely elevated (suprasystemic) pulmonary artery pressures, in addition to moderate right heart enlargement. This was in contrast to the neonatal intensive care unit (NICU) discharge echocardiogram from a month prior, where no ductus arteriosus was visualized and pulmonary pressures were estimated as only mildly elevated.

received

November 16, 2020

accepted after revision

February 10, 2021
DOI https://doi.org/

$10.1055 / \mathrm{s}-0041-1726462$

ISSN 2474-5871.
After admission, the patient remained on high ventilator settings requiring the addition of nitric oxide and had recurrent right upper lobe collapse likely from right atrial enlargement needing therapeutic bronchoscopy twice to maintain appropriate gas exchange. The patient continued to be sedated, paralyzed on mechanical ventilation, inhaled nitric oxide, diuretics, antibiotics, with multiple pulmonary hypertensive crises. The B type natriuretic peptide on admission was elevated at 2,252 picogram/milliliter (normal is $<100 \mathrm{pg} / \mathrm{mL}$ ) and improved to $242 \mathrm{pg} / \mathrm{mL}$ by day 5 , predicted time to diazoxide elimination. He was transitioned from nitric oxide to oral sildenafil (for ongoing pulmonary vasodilation) on day 8 and extubated on pediatric intensive care unit day 18. Diazoxide was discontinued since it was thought to be the inciting agent for the development of significant pulmonary hypertension ( $\mathrm{PH})$, but the patient continued to need high glucose infusion rates through total parenteral nutrition to maintain normoglycemia. Since the patient was critically ill and requiring a high glucose infusion rate, and

\section{(C) 2021. The Author(s).}

This is an open access article published by Thieme under the terms of the Creative Commons Attribution License, permitting unrestricted use, distribution, and reproduction so long as the original work is properly cited. (https://creativecommons.org/licenses/by/4.0/)

Georg Thieme Verlag KG, Rüdigerstraße 14, 70469 Stuttgart, Germany 
surgery was entertained as a possible option, a decision was made to obtain imaging with a Dopa positron emission tomography scan, which showed findings compatible with diffuse islet cell hyperplasia. A karyotype and chromosome microarray were normal $(\operatorname{arr}(1-22) x 2,(X Y) x 1)$. Methylation testing for Beckwith-Wiedemann syndrome [IC1 (H19) IC2 (LIT1)] was also normal. Rapid exome trio sequencing was completed and was nondiagnostic. Targeted ABCC8 sequencing with deletion/duplication testing identified only a single heterozygous missense variant of uncertain significance in the ABCC8 gene (c.1252T >C (p.Cys418Arg). This variant has been reported in both adult-onset diabetes and $\mathrm{HI}$, yet pathogenicity has not been fully established.

Due to the patient's abdominal distention and suspected necrotizing enterocolitis, treatment with octreotide was not thought to be a safe alternative because of potential for serious gastrointestinal side effects and also its risk for $\mathrm{PH}$. After a multidisciplinary discussion with $\mathrm{HI}$ experts due to the options of glucagon infusion gastrostomy tube with continuous glucose feeds, near total pancreatectomy, informed decision was made with endocrinology, cardiology, intensive care unit teams, and parenteral inclusion. After multiple discussions with parents, it was decided that definitive surgery with risk for complications was a last resort and instead a decision was made to restart the diazoxide 8 $\mathrm{mg} / \mathrm{kg} /$ day divided every 8 hours with a higher dose of chlorothiazide $20 \mathrm{mg} / \mathrm{kg} /$ day divided every 12 hours given the degree of his PH with close clinical and echocardiographic follow-up. The patient was discharged after being hospitalized for 57 days (last 3 weeks of hospitalization for feeding issues) with initiation of diazoxide $(8 \mathrm{mg} / \mathrm{kg} /$ day $)$ and chlorothiazide $20 \mathrm{mg} / \mathrm{kg} /$ day divided every 12 hours on day 22 with sequential echocardiograms obtained to ensure absence of $\mathrm{PH}$. Based on reassuring echocardiograms showing normalized pulmonary pressures and normal right ventricular size and function, sildenafil was discontinued on day 48 and the patient remained clinically well with normal subsequent echocardiograms. He has been followed outpatient now for 10 months post-discharge with no evidence of $\mathrm{PH}$ on echocardiograms and maintenance of normoglycemia on weaning doses of diazoxide. At 15 months of age, his fasting daily preprandial blood glucoses remained greater than 70 $\mathrm{mg} / \mathrm{dL}$ on diazoxide dose of $11.38 \mathrm{mg} / \mathrm{kg} /$ day and his diazoxide was successfully discontinued at that time. Blood glucose remained greater than $70 \mathrm{mg} / \mathrm{dL}$ after a 12-hour fast off treatment with diazoxide.

\section{Discussion}

Diazoxide is benzothiadiazide derivative previously studied as an antihypertensive medication and approved by the U.S. Food and Drug Administration (FDA) for use in children and infants for a specific subset of conditions, including symptomatic hyperinsulinemic hypoglycemia and the only therapy approved by the FDA for congenital HI. Diazoxide works by activation of the ATP-sensitive potassium channels. It suppresses insulin release from the pancreatic $\beta$-cell by maintaining a hyperpolarized plasma membrane hyperpolarized.
Review of the literature describes two distance categories of severe diazoxide-related adverse effects. One category includes the cardiac effects resulting from fluid retention and volume overload with subsequent congestive heart failure, ${ }^{1,2}$ and the other is that of distinct pulmonary hypertensive adverse effects. $^{3-6}$ The former phenomenon is much more frequently seen when diazoxide is given without concurrent administration of a thiazide diuretic to counteract the salt and water retention that are known side effects of diazoxide therapy alone. ${ }^{4}$ It is suspected that both side effects may be interrelated, in that the fluid overload to the right heart can exacerbate pulmonary hemodynamics and elevate pulmonary pressures, but it is not known whether diazoxide may have a direct effect on vasoconstriction of the pulmonary vasculature and thereby causing PH. In any event, both volume overload and $\mathrm{PH}$ were witnessed in our patient.

In September 2015, the FDA released a statement warning that $\mathrm{PH}$ had been reported in 11 cases of newborns and infant treated with diazoxide over many years. ${ }^{7}$ In retrospective studies of $\mathrm{HI}$ at congenital $\mathrm{HI}$ centers treated with diazoxide, development of $\mathrm{PH}$ with its use is a known but rare adverse event and after its initiation was observed in 2 to $4.8 \%$ of patients. $^{4,8,9}$ In addition to risk factors including prematurity, respiratory failure, and congenital heart disease, delayed chlorothiazide initiation was a risk factor for $\mathrm{PH}^{4}$ Most diazoxide-induced $\mathrm{PH}$ are thought to be reversible after discontinuation of diazoxide ${ }^{5,6}$ unless there is pre-existing $\mathrm{PH}^{4}{ }^{4}$ Cases of patent ductus arteriosus reopening have been reported, ${ }^{3}$ and sodium and water retention with an increase in pulmonary volume and pressures have been postulated to be related to reopening of the ductus arteriosus and worsening $\mathrm{PH} .{ }^{10}$

In infants, the incidence of $\mathrm{PH}$ may be higher as prevalence of diazoxide exposure in the NICU has increased along with the frequency of hypoglycemia as a diagnosis for infants in the NICU, doubling in the last 6 years being attributed to potentially the American Academy of Pediatrics in 2011 on criteria and treatment of hypoglycemia in newborns. ${ }^{11}$ Furthermore, an echocardiogram is not currently routinely ordered after the initiation of diazoxide. More recently it has been suggested that all infants being considered for diazoxide therapy receive an echocardiogram prior to and 5 days after initiation of diazoxide therapy, and continued thereafter as routine surveillance during the treatment. It has been also recommended that concurrent treatment with a thiazide diuretic be implemented. Also, parents should be informed about this possible side effect and to report any symptoms that might raise suspicion for $\mathrm{PH}$, such as respiratory distress, poor feeding, pallor, or cyanosis. ${ }^{6}$ It has been more recently found that serious adverse events including PH are significantly higher in newborns with perinatal stress HI than that of otherwise healthy babies with genetic forms of HI. This suggests that more caution should be used when prescribing diazoxide in this population ${ }^{8}$ as well as other drugs that have been associated with increased risk of PH in the newborn period. ${ }^{12}$

The mechanism of diazoxide-induced $\mathrm{PH}$ is unclear but direct toxic vascular reaction and KATP channel agonism have been postulated and it has been reportedly dose related. ${ }^{5,6}$ 
e82 Reintroduction of Diazoxide after Diagnosis of Pulmonary Hypertension in an Infant Schweiger et al.

\section{Conclusion}

Our case describes the successful reintroduction of diazoxide in an infant despite life-threatening $\mathrm{PH}$, undertaken due to lack of therapeutic options for congenital HI for this patient. This was achieved using a multidisciplinary approach involving cardiology, endocrinology, and the family, and undertaken using serial echocardiographic follow-up and close clinical surveillance.

\section{Authors' Contributions}

P.N. has made substantial contributions to the conception and design of the work; and helped in drafting the work and substantively revised it and has approved the submitted version and has agreed both to be personally accountable for the author's own contributions and to ensure that questions related to the accuracy or integrity of any part of the work, even ones in which the author was not personally involved, are appropriately investigated, resolved, and the resolution documented in the literature.

B.S. made substantial contributions to the conception and design of the work; and helped in drafting the work and substantively revised it and has approved the submitted version and has agreed both to be personally accountable for the author's own contributions and to ensure that questions related to the accuracy or integrity of any part of the work, even ones in which the author was not personally involved, are appropriately investigated, resolved, and the resolution documented in the literature.

\section{Funding}

None.

Conflict of Interest

None declared.

\section{References}

1 Silvani P, Camporesi A, Mandelli A, Wolfler A, Salvo I. A case of severe diazoxide toxicity. Paediatr Anaesth 2004;14(07):607-609

2 Welters A, Lerch C, Kummer S, et al. Long-term medical treatment in congenital hyperinsulinism: a descriptive analysis in a large cohort of patients from different clinical centers. Orphanet J Rare Dis 2015;10(01):150

3 Demirel F, Unal S, Çetin II, Esen I, Arasli A. Pulmonary hypertension and reopening of the ductus arteriosus in an infant treated with diazoxide. J Pediatr Endocrinol Metab 2011;24(78):603-605

4 Herrera A, Vajravelu ME, Givler S, et al. Prevalence of adverse events in children with congenital hyperinsulinism treated with diazoxide. J Clin Endocrinol Metab 2018;103(12):4365-4372

5 Nebesio TD, Hoover WC, Caldwell RL, Nitu ME, Eugster EA. Development of pulmonary hypertension in an infant treated with diazoxide. J Pediatr Endocrinol Metab 2007;20(08):939-944

6 Timlin MR, Black AB, Delaney HM, Matos RI, Percival CS. Development of pulmonary hypertension during treatment with diazoxide: a case series and literature review. Pediatr Cardiol 2017;38 (06):1247-1250

7 FDA Drug Safety Communication FDA Warns about a Serious Lung Condition in Infants and Newborns Treated with Proglycem (Diazoxide) [press release]. Food and Drug Administration. Maryland, USA: 2015

8 Thornton P, Truong L, Reynolds C, Hamby T, Nedrelow J. Rate of serious adverse events associated with diazoxide treatment of patients with hyperinsulinism. Horm Res Paediatr 2019;91(01): 25-32

9 Gray KD, Dudash K, Escobar C, et al; Best Pharmaceuticals for Children Act-Pediatric Trials Network Steering Committee. Prevalence and safety of diazoxide in the neonatal intensive care unit.] Perinatol 2018;38(11):1496-1502

10 Pediatrics AAo. Postnatal glucose homeostasis in late-preterm and term infants. Pediatrics 2011;127(03):575-579

11 Silvani P, Camporesi A. Drug-induced pulmonary hypertension in newborns: a review. Curr Vasc Pharmacol 2007;5(02):129-133

12 Yildizdas D, Erdem S, Küçükosmanoglu O, Yilmaz M, Yüksel B. Pulmonary hypertension, heart failure and neutropenia due to diazoxide therapy. Adv Ther 2008;25(05):515-519 